\title{
Factors affecting decisions to accept or decline cystic fibrosis carrier testing/screening: A theory-guided systematic review
}

\author{
Lei-Shih Chen, PhD, CHES ${ }^{1}$, and Patricia Goodson, $\mathrm{PhD}^{2}$
}

\begin{abstract}
Purpose: To assess factors affecting individuals' decision to accept or decline cystic fibrosis carrier testing/ screening, as reported in studies published until December 2006. The Health Belief Model guided classification of each factor, and the studies' methodological quality was assessed. Methods: A three-stage search and retrieval process, alongside application of specific inclusion/exclusion criteria, yielded 40 studies (in 35 articles). For each reviewed study, authors abstracted and organized selected data into a matrix and assigned a methodological quality score. Results: The four most frequently identified acceptance factors included three Health Belief Model factors and one non-Health Belief Model factor: perceived benefits of undergoing cystic fibrosis carrier testing/ screening, weaker perception of barriers to cystic fibrosis carrier testing/screening, fewer/no children or desiring children, and research-related factors (non-Health Belief Model factor construct). All four most frequent factors associated with declining testing were Health Belief Model factor constructs: perceived barriers to obtaining cystic fibrosis carrier testing/screening, parity, lack of knowledge, and weaker perception of benefits of undergoing cystic fibrosis carrier testing/screening. The average methodological quality of the studies was 10.2 (SD = 3.2; range, 5-18 points). Conclusions: The methodological and theoretical quality of this body of literature could be substantially improved if researchers employed theory-based approaches, tested (and reported) the validity/reliability of their own data, and employed multivariate statistical analyses and/or better controlled research designs. Improving the quality of future studies may allow better inferences regarding the relative contribution of each factor identified in this review to individuals' decision-making process. Genet Med 2007:9(7):442-450.
\end{abstract}

Key Words: cystic fibrosis, factors, carrier testing, carrier screening, systematic review

As the most frequent autosomal recessive disease among whites, cystic fibrosis (CF) affects nearly one in 2500 livebirths within this group, and approximately 1 in 25 white individuals are carriers. ${ }^{1}$ After scientists identified the CF gene in 1989 and developed sensitive tests (able to detect mutations associated with CF among 75-95\% of carriers ${ }^{2,3}$ ), carrier testing/screening became feasible and an important aid in preventing or managing CF. ${ }^{4}$ Carrier testing for CF provides individuals with relevant information for family planning and for the purpose of preventing or preparing for an affected child because early diagnosis can reduce medical costs and lessen parents' suffering due to uncertainty. ${ }^{5}$

Even though professional organizations have recommended $\mathrm{CF}$ carrier screening and providers have made testing a routine

From the ${ }^{1}$ Department of Public Health, University of North Florida, Jacksonville, Florida; and ${ }^{2}$ Department of Health and Kinesiology, Texas A\&M University, College Station, Texas. Disclosure: The authors declare no conflict of interest.

Patricia Goodson, PhD, Department of Health and Kinesiology, 4243 TAMU, Texas AઐM University, College Station, TX 77843-243; E-mail:pgoodson@hlkn.tamu.edu

Submitted for publication January 8, 2007.

Accepted for publication April 24, 2007.

DOI: 10.1097/GIM.0b013e3180986767 obstetric practice since $2001,{ }^{6-8}$ uptake of the test remains relatively low, especially for preconception purposes. Murray et al. ${ }^{5}$ documents an average acceptance rate for prenatal CF carrier testing of 74\% (range, 59-99\%) after reviewing 11 studies. For preconception use, however, we find an acceptance rate of $47.6 \%$, varying from $8 \%$ to $76 \%$ among different studies. ${ }^{9-12}$

To help develop cost-effective CF carrier screening and educational programs, factors affecting individuals' decision to undergo or forgo testing must be identified and well understood. For this purpose, scientific literature reviews represent helpful tools as they assess the extant studies focusing on such factors. Decruyenaere et al. ${ }^{1}$ published the most recent effort of this sort in 1998. Their review had multiple aims: to examine the research regarding the uptake of CF testing and its impact on self-esteem, risk perception, and reproductive decisions; to relate study findings to "psychological theories and concepts"; and to "infer practical suggestions for genetic counseling."

Although the review by Decruyenaere et al. ${ }^{1}$ deserves commendation for identifying the need for theory within studies of genetic carrier testing/screening, it failed to employ systematic review methods (e.g., authors provide no description of inclusion/exclusion criteria) or to evaluate the methodological quality of the findings it summarized. Our report, therefore, 
focuses on systematically assessing and critically examining the data regarding factors affecting individuals' decisions to accept or decline CF carrier testing/screening.

We employed systematic literature review methods ${ }^{13}$ to accomplish our purpose because these methods have been found to strengthen "the link between best research evidence and optimal health care" and to provide "synthesis of best evidence for clinical decisions." ${ }^{14}$ Moreover, this systematic review adds two important dimensions, normally absent from traditional syntheses of the literature: we employed a theoretical framework (the Health Belief Model [HBM]) to organize individual findings according to theoretically identified categories, and we assessed the methodological quality of each study, according to 11 criteria.

Specifically, this systematic review answers four questions: (1) What are the factors affecting individuals' decision to accept CF carrier testing/screening? (2) What are the factors affecting individuals' decision to decline CF carrier testing/ screening? (3) Do the factors influencing individuals' decision making regarding acceptance or decline of CF carrier testing differ in important ways? (4) What is the methodological quality of the body of literature examining CF carrier screening decision making?

For this review's purposes, we defined acceptance factors as variables associated with (a) study participants actually undergoing CF carrier testing; (b) participants' decision to undergo testing; (c) participants' positive (or accepting) attitudes toward testing; and (d) their expressed likelihood or intention of undergoing testing (in hypothetical scenarios). Conversely, decline factors referred to these same dimensions described for acceptance, but focused on the avoidance of testing or refusal to test.

It is important to note that we do not distinguish a priori between factors associated with carrier testing in prenatal or preconception time frames, as such a distinction would yield smaller numbers of studies reviewed under each category. When discussing individual studies' findings, however, we mention whether the research was performed with pregnant or nonpregnant women when the reviewed studies provided the information. Finally, although scholars and practitioners distinguish between the terms "testing" and "screening"- the former referring typically to the testing of individuals with a specific family history and the latter to the screening of individuals in an at-risk population, ${ }^{15}$ we found that only six reviewed studies assessed CF carrier testing. ${ }^{2,16-20}$ The majority assessed carrier screening $(n=34)$. Given the small number of studies in the testing category, we did not analyze them separately. Additionally, in this report, we use both terms interchangeably, reflecting the manner in which the study authors themselves often fail to discriminate between the terms. For a clear example of this difficulty, see Mennie et al. ${ }^{21}$

\section{Theoretical framework: the Health Belief Model (HBM)}

Using a theoretical framework to organize findings from various studies represents an advantage over traditional sys- tematic reviews: as theory identifies both the constructs and the logical connections (or hypothetical relationships) among constructs, it facilitates causal (logical) thinking and knowledge development. Using theoretical categories also helps identify which factors are over- or underrepresented in the body of literature under review. ${ }^{22-24}$

For this review, we chose to employ the HBM as the organizing theoretical framework because it is well suited for understanding screening behaviors. The model has a unique historical development as it "grew out of practical concerns with the limited success of various programs of the U.S. Public Health Service in the 1950s."25 One instance of such "limited success" was a free tuberculosis x-ray screening campaign for adults. The HBM developed, thus, as an attempt to explain people's behavior in avoiding being screened. Ever since, the HBM has been successfully used to explain numerous other health behaviors aside from uptake of screening tests, but its historical origins and application make it especially useful in understanding decision making regarding CF testing.

The HBM includes intrapersonal constructs such as perceived benefits, perceived barriers, perceived severity of an illness, one's perceived susceptibility to a disease, and environmental and/or psychosocial cues to action. The model also includes modifying factors, such as knowledge, ethnicity, marital status, number of children, and socioeconomic status. Modifying factors (commonly demographic characteristics, personality traits, cognitive ability) moderate the relationships occurring between specific intrapersonal constructs and the decision to engage in or avoid a particular health behavior.

\section{METHODS}

\section{Literature search}

A three-step procedure guided the literature search. First, we attempted to retrieve all peer-reviewed journal articles investigating factors influencing CF carrier testing/screening decision making from 14 electronic databases (e.g., Medline, ERIC, PsychINFO, Annual Reviews, and CINAHL). We employed variations and Boolean connections of the key terms decision making, attitude, genetic screening, carrier screening, carrier testing, carrier, and cystic fibrosis. This search retrieved 183 reports from the earliest years of the databases to December 2006, by limiting the search to English-language publications.

The second step involved assessing which studies met selected inclusion/exclusion criteria. Studies met the inclusion criteria if they (1) appeared in a peer-reviewed, English-language journal; (2) reported original studies; (3) empirically examined factors affecting individuals' decision making regarding CF carrier testing/screening; and/or (4) directly assessed attitudes toward testing/screening among individuals who underwent testing. The exclusion criteria helped eliminate (1) theoretical, nonempirical, or review studies; (2) published abstracts; (3) reports that did not explore factors affecting $\mathrm{CF}$ decision making regarding testing/screening; (4) studies comparing the results from different CF testing/screening approaches, such as contrasting findings between stepwise 
screening and couple screening; and/or (5) articles reporting people's attitudes toward testing of others, not themselves (e.g., asking wives whether they believed their husbands should be tested). Twenty-four studies (of the original 183) met the inclusion and exclusion criteria.

In the third step, we reviewed these 24 studies' reference lists carefully for additional publications; moreover, we searched for additional publications using the first and last author names listed in the 24 retrieved studies, using the ISI Web of Knowledge database. Eleven additional studies retrieved in this phase met the inclusion/exclusion criteria $(N=35)$. As 5 of these 35 studies used two approaches to examine factors for $\mathrm{CF}$ carrier testing/screening, we considered each approach as a separate study, yielding a final sample size of 40 studies from 35 reviewed articles.

\section{Collecting and organizing data from each study}

To systematically structure and organize the information collected from each study, the authors developed a review matrix (due to its length, the full matrix is not included here but is available upon request to the authors). This matrix allowed the authors to abstract information on study design, sample characteristics, data collection methods, measurement instruments, reporting of data validity and reliability, statistical techniques employed in data analyses, study findings (acceptance and decline factors), and overall methodological quality score. ${ }^{13}$

Readers should bear in mind that when collating information regarding measurement instruments and reporting of data validity/reliability, we focused on factors affecting decisions to accept or decline CF testing/screening exclusively. For example, if a study measured both knowledge and attitudes regarding CF carrier testing, we only collected information regarding measurement instruments and reporting of validity/ reliability for the attitude variable.

When organizing studies' findings of factors associated with CF carrier testing among studies employing only descriptive statistics (such as frequency distributions), factors for which authors provided any descriptive data were considered valid. When studies employed statistical testing, we opted for reporting only factors resulting from analyses with the maximum amount of statistical control. For instance, if findings comprised $\chi^{2}$ analyses and multiple regression coefficients associated with specific factors, we counted as valid only the factors for which statistically significant regression coefficients were reported. Additionally, when abstracting study findings, we employed a more conservative level of probability for statistical testing $(P<0.05)$, even though some studies used higher probability levels, such as 0.10 , for their critical values. Both authors abstracted the information from 10 studies (30\%) independently to assess the reliability of the coding or data abstraction process. An average interrater reliability index, calculated across these 10 studies, was 0.80 (Cohen's $\kappa ; 70 \%$ of scores ranged from 0.86 to 1.00 ).

\section{Methodological Quality Score (MQS)}

To assess the methodological characteristics of this body of knowledge, we rated each individual study using an MQS. The criteria for the MQS, adapted from previous reviews to include criteria most appropriate for CF screening/testing studies, ${ }^{26,27}$ include assessments of each study's design, its use of theory, sample size, reporting of sample characteristics (such as age and ethnicity), the use of previously tested measures, testing and reporting of the study data's validity and reliability, level of sophistication of the analytical techniques, and use of statistical controls when determining the association between specific factors and individuals' decision regarding CF testing/screening. Table 1 lists the frequency distributions of each element of the MQS criteria for the 40 reviewed studies along with the scoring criteria. Possible MQSs range from 4 to 20 points. A higher MQS represents better methodological quality.

\section{RESULTS}

\section{Studies' characteristics}

Forty studies (published in 35 manuscripts) met our inclusion/exclusion criteria. These included 38 studies with a quantitative, survey-type, convenience sample design and two studies with a qualitative design. Studies originated in the United States (17), United Kingdom (9), Belgium (5), Canada (4), The Netherlands (3), and Australia (2) and were published between 1989 and 2004, with the largest number of reviewed publications $(n=20)$ found between 1995 and 1999.

\section{Studies' findings}

Below we present studies' findings categorized by each of the HBM constructs (see "Theoretical framework"). The few factors that could not be classified as HBM elements are identified as non-HBM factors. Due to space limitations, we discuss thoroughly the four most frequently identified factors (in decreasing order of frequency) and list those occurring less frequently. We do not discuss factors documented in $<10 \%$ of studies $(n<$ 4 ), but identify them in the review matrix (available upon request).

\section{Factors affecting decisions to accept CF carrier testing/screening}

\section{Perceived benefits of undergoing CF carrier testing/screening}

Fourteen reviewed studies (35\%) identified individuals who strongly perceived the benefits of undergoing CF carrier testing/screening as more likely to be tested. Three studies using the HBM as a theoretical framework directly assessed the construct perceived benefits. ${ }^{28-30}$ Fang et al., ${ }^{28}$ for example, when evaluating a hypothesized path model, found that perceptions of greater benefits from screening predicted more positive attitudes toward CF testing.

Among these 14 studies reporting perceived benefits as a factor influencing CF carrier testing, knowing one's carrier status and not having an affected child were the two most often recognized benefits of testing. For example, Delvaux et al. ${ }^{3}$ of- 
Table 1

Methodological criteria and frequency distribution of each criterion among 40 reviewed studies

\begin{tabular}{|c|c|c|c|}
\hline \multirow[b]{2}{*}{ Methodological characteristic } & \multirow[b]{2}{*}{ Scoring options (maximum total score $=20$ points) } & \multicolumn{2}{|c|}{$\begin{array}{l}\text { Distribution of } \\
\text { characteristics } \\
\text { among } 40 \text { reviewed studies }\end{array}$} \\
\hline & & $\begin{array}{l}\text { Frequency } \\
\qquad(n)\end{array}$ & $\%$ \\
\hline \multirow[t]{3}{*}{ Study design } & Correlation $/$ cross-section design $=1$ point & 37 & 92.5 \\
\hline & Retrospective design $=2$ points & 0 & 0 \\
\hline & Prospective design $=3$ points & 3 & 7.5 \\
\hline \multirow[t]{3}{*}{ Theoretical framework for quantitative studies ${ }^{a}$} & Study had no theory $=0$ points & 24 & 63.2 \\
\hline & Study was based on an implicit theory $=1$ point & 10 & 26.3 \\
\hline & Study was based on a specific theory $=2$ points & 4 & 10.5 \\
\hline \multirow[t]{3}{*}{ Theoretical framework for qualitative studies ${ }^{b}$} & $\begin{array}{l}\text { Study neither built a theory nor linked its findings to a } \\
\text { specific theory }=0 \text { points }\end{array}$ & 2 & 100 \\
\hline & Study linked its findings to a specific theory $=1$ point & 0 & 0 \\
\hline & The study developed a theory $=2$ points & 0 & 0 \\
\hline \multirow[t]{3}{*}{ Sample size } & Small sample $(<100)=1$ point & 6 & 15 \\
\hline & Medium sample $(>100$ and $<300)=2$ points & 13 & 32.5 \\
\hline & Large sample $(>300)=3$ points & 21 & 52.5 \\
\hline \multirow[t]{2}{*}{ Age } & Not reported $=0$ points & 7 & 17.5 \\
\hline & Reported $=1$ point & 33 & 82.5 \\
\hline \multirow[t]{2}{*}{ Ethnicity } & Not reported $=0$ points & 22 & 55 \\
\hline & Reported $=1$ point & 18 & 45 \\
\hline \multirow[t]{2}{*}{ Measurement instrument ${ }^{c}$} & $\begin{array}{l}\text { Authors developed the instrument measuring decision-making } \\
\text { factors }=1 \text { point }\end{array}$ & 34 & 85 \\
\hline & $\begin{array}{l}\text { Authors adopted a previously established instrument measuring } \\
\text { decision-making factors }=2 \text { points }\end{array}$ & 6 & 15 \\
\hline \multirow[t]{2}{*}{ Data validity testing } & Not reported $=0$ points & 35 & 87.5 \\
\hline & Reported $=1$ point & 5 & 12.5 \\
\hline \multirow[t]{2}{*}{ Data reliability testing } & Not reported $=0$ points & 30 & 75 \\
\hline & Reported $=1$ point & 10 & 25 \\
\hline \multirow[t]{5}{*}{ Data analysis ${ }^{d}$} & $\begin{array}{l}\text { Qualitative analyses (content analysis and thematic analysis) }=1 \\
\text { point }\end{array}$ & 2 & 5 \\
\hline & Univariate statistics/descriptive $=1$ point & 12 & 30 \\
\hline & Bivariate statistics/ANOVA $=2$ points & 16 & 40 \\
\hline & Logistic regression/ANCOVA $=3$ points & 9 & 22.5 \\
\hline & Multivariate statistics (structural equation modeling) $=4$ points & 1 & 2.5 \\
\hline \multirow[t]{3}{*}{ Factors for CF carrier testing/screening } & No factors were identified $=0$ points & 2 & 5 \\
\hline & Factors were not tested for statistical significance $=1$ point & 18 & 45 \\
\hline & Factors were tested for statistical significance $=2$ points & 20 & 50 \\
\hline
\end{tabular}

ANOVA, analysis of variance; ANCOVA, analysis of covariance.

${ }^{a}$ The percentage was calculated based on 38 quantitative studies.

${ }^{b}$ The percentage was calculated based on two qualitative studies.

'If the study did not cite any references regarding their instrument, we assumed they developed the instrument themselves.

${ }^{d}$ Studies were given credit for the most sophisticated statistical analysis employed, regardless of whether the more robust analysis was used to examine acceptance/ decline factors or to assess other variables (e.g., knowledge). 
fered CF carrier screening (not free) to couples undergoing prenatal testing for non-CF-related issues. Three months after learning their test result, more than one third of 400 participants claimed knowing their carrier status and avoiding a child with $\mathrm{CF}$ as the main reasons leading them to undergo testing. Similarly, in the study conducted by Hartley et al., ${ }^{18} 623$ pregnant women ( $<14$ weeks' gestation) received a leaflet regarding CF carrier testing and were asked whether they wanted to be tested. A total of 529 (84.9\%) women accepted the test. For these women, either wanting to know whether they were a carrier of the CF gene or being certain they did not want to have an affected child constituted the main factor(s) affecting their decision to accept CF prenatal carrier testing.

\section{Weaker perception of barriers to CF carrier testing/screening}

Eight reviewed studies (20\%) indicated that fewer perceived barriers to obtaining the CF carrier test were associated strongly with its acceptance. Three studies ${ }^{29-31}$ documented that individuals who perceived few barriers to undergoing testing had more positive attitudes toward the test. In the study by Henneman et al., ${ }^{30}$ for example, general practitioners invited couples considering a future pregnancy and interested in preconception CF screening, to attend a 45-minute educational session. Seventy-six couples attended and completed a survey assessing knowledge and attitudes regarding screening. Fiftythree couples neither interested in testing nor attending the educational session also completed the survey. Couples who did not perceive potentially negative consequences from testing (such as social stigma) as barriers, were seven times more likely to accept screening than those who viewed such potential outcomes as significant obstacles.

Low tolerance for test uncertainty and cost of the (CF carrier) test represented two major obstacles for individuals to accept testing. Tambor et al. ${ }^{32}$ carried out two studies (mail invitation approach and on-site invitation approach) to survey primarily nonpregnant health maintenance organization (HMO) enrollees' attitudes. Both studies demonstrated higher tolerance for uncertainty as associated with higher likelihood of test uptake. Another study conducted by Durfy et al. ${ }^{33}$ also reported acceptance of CF carrier testing as inversely related to its cost (free or low cost).

\section{Fewer/no children, or desiring children}

Having fewer/no children or desiring children constituted the second most cited factor affecting individuals' acceptance of CF carrier screening (identified in eight studies; 20\%). Among these studies, five indicated that women without children would be more likely to undertake the test. In the study by Witt et al., ${ }^{34}$ for instance, providers offered CF heterozygote screening to 6617 primarily white or Hispanic pregnant women during a routine HMO parental class. A total of 5161 (78\%) women agreed with testing (consenters), 947 (14\%) declined the test but completed the survey (decliners), and 509 $(8 \%)$ neither accepted the test nor completed the survey. When comparing consenters' and decliners' characteristics, consenters were more likely to be nulliparous $\left(\chi^{2} ; P<0.001\right)$.

Desire for children also affected CF carrier testing/screening decision making. Honnor et al. ${ }^{12}$ provided pre-education questionnaires, information leaflets, and posteducation questionnaires to 5102 English-speaking individuals attending one family planning clinic and 20 general practices in Australia. Individuals with a stronger intention to have children were almost twice as likely to have been tested as those who had weaker intentions.

\section{Research-related factors (non-HBM)}

Eight of the 40 reviewed studies (20\%) also indicated research-related factors as important reasons for seeking CF carrier testing/screening. These factors included a desire to assist with the medical research being conducted, a particular health care provider's influence, and the client's perceived difficulty or inability to refuse the offer to test. According to the studies carried out by Harris et al. ${ }^{35}$ Hartley et al., ${ }^{18}$ and Delvaux et al., ${ }^{3}$ all of which employed health care providers to explain the research project to participants, pregnant women claimed "feeling they could not refuse the (CF carrier) test" as one of the main reasons for agreeing with testing. In addition, Poppelaars et al. ${ }^{36}$ indicated that recently married couples who felt "high perceived pressure from experts" were more likely to accept preconceptional CF carrier testing. As illustrated by the statement from one couple who was tested subsequent to their midwife's suggestion, "The midwife urged us to have the test, but we didn't really want to. We planned to go on holiday two days later...."20

\section{Other factors affecting decisions to accept CF carrier testing/screening}

Below we list other CF carrier testing/screening acceptance factors occurring less frequently in this literature (but identified in at least $10 \%$ of reviewed studies). We list each factor along with its frequency among reviewed studies.

- High socioeconomic status (HBM modifying factor; $17.5 \%)$ : higher educational level, and higher income were associated with acceptance of CF testing. 2,12,28,34,37,38

- Strong perceived susceptibility of being a CF carrier (HBM factor; 17.5\%): Mélancon and De Braekeleer ${ }^{39}$ found that "presence of a hereditary disorder in the family" would affect adolescents' attitudes toward accepting CF carrier screening.

- Ethnicity (HBM modifying factor; 12.5\%): whites were more likely to accept carrier testing than nonwhites. . $^{32,34,37,38}$

- More CF-related knowledge (HBM modifying factor; $12.5 \%$ ): Clayton et al..$^{37}$ found that people with previous CF-related knowledge opted to obtain CF carrier testing/ screening.

- Gender (HBM modifying factor; 12.5\%): Four studies in three articles ${ }^{2,12,40}$ indicated that being female correlated with acceptance of CF carrier testing, whereas one study ${ }^{37}$ documented the opposite association. 
- Strong perceived severity of being a CF carrier (HBM factor; $10 \%$ ): Three studies (in two publications) $)^{4,36}$ indicated that stronger perceptions of the severity of being a carrier were associated with a higher likelihood of accepting CF carrier testing. Yet, one study ${ }^{29}$ indicated that people with a high perceived severity of being a carrier tended to decline the test.

- Age (HBM modifying factor; 10\%): the four studies identifying this factor exhibited inconsistent results. ${ }^{12,29,32,41}$

- Personality (non-HBM factor; 10\%): according to Henneman et al., ${ }^{30}$ individuals with a higher internal locus of control tended to better accept CF carrier testing than those with less sense of control over their own health.

\section{Factors affecting decisions to decline CF carrier testing/screening}

\section{Perceived barriers to obtaining CF carrier testing/screening}

We identified perceived barriers to obtaining a CF carrier testing/screening as the most frequent factor associated with decisions to decline testing (found in seven reviewed studies; $17.5 \%)$. The three studies assessing this HBM construct ${ }^{28-30}$ documented perceptions of barriers to testing as positively associated with refusal to test.

Fear of a blood test and lack of time represented two specific perceived barriers affecting individuals' decision to decline. For example, Mitchell et al. ${ }^{42}$ and Durfy et al. ${ }^{33}$ conducted two separate studies to evaluate high school students' attitudes toward CF carrier testing. Fear of the blood test was recognized as a factor leading to refusal in both studies. Additionally, Payne et al. ${ }^{43}$ provided population CF carrier screening to 1553 nonpregnant patients in one general practice in South Wales in the United Kingdom. Among the 65 untested patients, nearly half stated that they forgot the appointment or did not have time to undergo testing.

\section{Parity}

Parity was also the most frequently identified factor for people refusing CF carrier testing ( $n=7,17.5 \%$ of reviewed studies). Six studies ${ }^{4,12,34,38,44}$ indicated that having children or more children was positively associated with declining testing. When comparing pregnant women who accepted testing to those who refused the screening for example, Mennie et al. ${ }^{44}$ found that multiparous women tended to decline the CF test more often than primiparous women. Similarly, Loader et al. ${ }^{38}$ also identified that higher numbers of pregnant women who declined testing already had children. Potentially, having had CF-free offspring may have led these women to perceive themselves at lower risk. ${ }^{44}$

Although not planning for more children was a factor influencing individuals' decisions related to CF carrier testing, we encountered inconsistent findings. Studies conducted by Denayer et al. ${ }^{16}$ and Honnor et al. ${ }^{12}$ found among individuals who did not plan for more children a higher likelihood of declining CF carrier testing, whereas Payne et al..$^{43}$ documented the opposite.

\section{Lack of knowledge}

Lack of CF-related knowledge also affects individuals' refusal to test. Six reviewed studies in four publications $(15.0 \%)^{4,30,37,40}$ reported clients with less CF-related knowledge as having a higher probability of declining the test, whereas only one study ${ }^{45}$ reported the opposite finding. In the research of Clayton et al., ${ }^{37}$ for example, 238 nonpregnant adults reviewed CF-related educational materials and completed questionnaires examining factors affecting their decision making. Compared with those who submitted blood samples for carrier testing, those who refused did not have similar previous knowledge about CF. When compared with individuals who had previously had CF carrier testing, Lafayette et al. ${ }^{40}$ indicated that those who had not previously been tested scored significantly lower on CF-related knowledge survey items. Botkin and Alemagno, ${ }^{45}$ however, found that pregnant women who answered questions regarding the nature of $\mathrm{CF}$ correctly were less likely to undergo CF carrier testing during pregnancy.

\section{Weaker perception of benefits of undergoing CF carrier testing/screening}

Five reviewed studies $(12.5 \%)^{4,29,30,37,46}$ reported weaker perceptions of the benefits of testing as associated with the decline of CF carrier screening. O'Connor and Cappelli ${ }^{29}$ surveyed 131 adults who accompanied children to an orthopedic outpatient clinic. When compared with those considering CF carrier screening, those who would not consider screening had a lower score in the "perceived benefits of obtaining screening" survey items. Tatsugawa et al. ${ }^{46}$ and Levenkron et al. ${ }^{4}$ ascertained that women who did not feel CF carrier testing information was necessary for their pregnancy did not believe that women should be offered CF carrier screening or would not recommend $\mathrm{CF}$ carrier screening to a female friend were more inclined to reject the CF carrier testing offer.

\section{Other factors affecting decisions to decline CF carrier testing/screening}

Factors associated with decisions to refuse CF carrier testing/screening reported less frequently yet encountered in at least $10 \%$ of reviewed studies included:

- Perceived severity of being a CF carrier (HBM factor; 10\%): two studies ${ }^{47}$ indicated that strong perceptions of the severity of being a carrier correlated with the refusal to undergo testing, whereas two other studies reported by Levenkron et al. ${ }^{4}$ exhibited opposite findings.

- Weak perceived susceptibility of being a CF carrier (HBM factor; 10\%): Cuckle et al. ${ }^{48}$ found that pregnant women refused to be tested because they did not feel that CF was a common disease.

- Attitudes against abortion (non-HBM factor; 10\%): women who were against termination of an affected pregnancy had a negative attitude toward CF carrier testing in three studies. ${ }^{4,48,49}$ 
- Ethnicity (HBM modifying factor; $10 \%$ ): nonwhites were more likely to decline testing. ${ }^{34,37,38}$

- Low socioeconomic status (HBM modifying factor; 10\%): lower educational levels and lower income were associated with refusal. ${ }^{12,34,37,38}$

- Personality (non-HBM factor; 10\%): Fang et al..$^{28}$ showed that avoidant coping factors such as distancing and denial were indirectly associated with pregnant women's rejecting carrier testing.

\section{Methodological quality of studies}

The methodological quality of the reviewed studies varied. The average MQS was $10.2(\mathrm{SD}=3.2)$, within a theoretical range of 4 to 20 points (actual range, 5-18). Most reviewed studies $(92.5 \%)$ comprised cross-sectional designs and more than half $(52.5 \%)$ examined large (>300 respondents) yet nonprobability samples. The majority of studies were not theory based (63.2\% of quantitative studies). Among those that employed a theoretical framework, three studies used the HBM $(n=3)$ and one applied the theory of cognitive dissonance $(n=1)$ to guide the inquiry.

Regarding reporting of study sample characteristics such as age and ethnicity, most reported age of participants (82.5\%), but ethnicity was not described among $55 \%$ of reviewed studies. Most researchers (85\%) developed their own measures of the decision-making factors they examined, but failed to report tests of their data's validity and reliability: only $12.5 \%$ reported data validity and 25\% reported reliability. Among quantitative studies, $70 \%$ used univariate/bivariate statistics to analyze their data. Only one study ${ }^{28}$ used structural equation modeling to assess factors related to decision making. Half of the reviewed studies did not submit the factors they identified as associated with decisions to accept or decline to any inferential statistical tests (e.g., statistical significance).

\section{DISCUSSION}

In answering the first two proposed questions - what are the factors affecting an individual's decision to accept or decline CF carrier testing/screening?- - this review identified 13 factors associated with acceptance or refusal of CF carrier testing/ screening. The four most frequent acceptance factors included (in order of frequency): perceived benefits of undergoing CF carrier testing/screening, weaker perception of barriers to obtaining CF carrier testing/screening, having fewer/no children or desiring children, and research-related factors. The four most frequent factors associated with declining CF carrier testing included perceptions of barriers to obtaining CF carrier testing/screening, parity, lack of CF-related knowledge, and weaker perception of the benefits of being tested.

The third question that this review set out to answer focused on whether important differences existed between these two sets of factors. It was interesting to note that among the 13 factors correlated with acceptance or decline, 9 were common to both behaviors, appearing, nevertheless, with different frequencies. Also worthy of notice was the identification of atti- tudes toward abortion as a factor associated exclusively with nonconsenting decisions and research-related factors, gender, and age as associated only with acceptance of testing.

These findings suggest that although some factors remain common, they vary in relative importance when associated with accepting or declining behaviors and should prompt professionals developing educational or testing programs to consider them individually. To assume that the most important (most frequent) rationale associated with accepting testing represents merely the inverse of the most salient motivation for refusing the test can be problematic. For example, to conclude that because stronger perceptions of the benefits of testing are associated with acceptance, weaker perceptions of benefits will automatically be associated with decline, may lead to educational messages that overemphasize benefits in order to increase test uptake. Such overemphasis may not take into consideration that, according to the literature reviewed here, perceptions of barriers to testing occur more frequently as a reason for decline (and are perhaps more salient) than weak perceptions of benefits.

Educational materials and/or programs alongside health care providers who deal with CF carrier testing/screening would do well to regard "acceptors" and "decliners" as unique groups and to design interventions taking into account the issues most pertinent to each (starting where the clients are). Furthermore, additional studies designed to assess the relative contribution of each of these identified factors are sorely needed. Most of the research reviewed here, descriptive in nature, does not allow appropriate comparisons or statistical controlling of variables to determine each factor's weighted contribution to an individual's decision making.

Last, research scholars and health care providers must continue to develop better understanding of the mechanisms linking specific factors and individuals' decisions whether to undergo testing. Perceived severity of being a carrier, for instance, was associated (albeit less frequently) with both acceptance and decline. Further studies are needed to better comprehend under which circumstances, and for what types of clients, perceptions of the severity of a carrier status function as a facilitator or a deterrent to screening.

Of particular concern was finding that research-related issues (e.g., study participants acknowledging acceptance of the test due to perceived pressure from health care providers) constituted a factor associated with acceptance of carrier testing among $20 \%$ of the reviewed studies. It was not clear, however, whether pressure indeed was exerted or whether clients' perceptions construed the offer to screen as part of a research project as a "push" for acceptance. Although offered as both a prevention and uncertainty-management tool, CF carrier testing nonetheless comprises many complex ethical, legal, and social issues, including the potential for social stigma, peer pressure, and genetic discrimination. Therefore, care should be taken to ensure that an individual's reproductive autonomy is respected and informed decision making is the primary goal of screening programs. While true nondirectiveness in the case of carrier screening/testing is extremely difficult to achieve in practice, researchers and health care providers should be espe- 
cially sensitive to the strategies employed to inform individuals of the availability of CF screening/testing and to the manner in which they facilitate their clients' informed decision making.

The fourth and final question that this review proposed to answer relates to the overall methodological quality of this body of knowledge. The mean MQS of 10.2 indicates these studies are, as a whole, of average quality (relative to our criteria), whereas a pattern of important weaknesses emerges from this analysis. The first weakness of this literature comprises the paucity of theoretically driven or theoretically operationalized research assessing individuals' decision making regarding CF carrier testing. Only 4 of the 40 reviewed studies alluded to a health behavioral or psychological theory, and only 2 different theories (i.e., the HBM and the theory of cognitive dissonance) were referenced. Such small sample and the equally small number of theories allow no conclusions, at this point, regarding which theory provides the best articulation of factors associated with an individual's decision making regarding CF carrier testing. Our use of the HBM in this review suggests the model may provide adequate explanatory factors for understanding an individual's decision to accept or decline testing. The fact that we identified several elements that could not be neatly classified as HBM constructs, however, raises the question of whether the HBM remains the most appropriate theoretical approach. Additional studies seeking to contribute to the theoretical understanding of these factors are certainly needed.

A second weakness in this group of studies is the absence of reporting of study data validity and reliability. Without analyzing data validity and reliability, consumers cannot have confidence in the validity of the findings or in their replicability due to the nontrivial increase in measurement error. ${ }^{50}$

A third common weakness regards descriptions of the samples' ethnic characteristics: although whites are the primary target population for $\mathrm{CF}$ carrier screening, describing participants' ethnicity is vital, especially because ethnicity is itself a factor associated with acceptance among $12.5 \%$ of reviewed studies. Unfortunately, more than half of reviewed studies were unclear about participants' ethnic backgrounds.

A fourth and final weakness encompasses study design: employing more sophisticated research designs and data analytic techniques would substantially enhance the quality of this body of knowledge and allow better inferences regarding the relative contribution of each factor to an individual's decisionmaking process. Researchers in this field should be challenged to develop better measures and better analytical tools to account for the multivariate nature of human (and biological) reality, the shared variance existing among factors, and the inevitable interactions among factors.

Yet even as this review provides an assessment of the overall quality of this body of research, it exhibits important limitations of its own. One such limitation was our inability to review all the relevant literature on CF carrier testing. This was due primarily to our restricting the search to articles published in English. Our search strategy may also have missed important studies that were not indexed in the databases searched. We attempted to overcome the latter limitation by combining various search strategies.

Moreover, as there is no gold standard for judging the methodological quality of survey studies, especially when research involves nonexperimental designs, our criteria for developing the MQS were self-referential and were biased toward more rigorous, experimental-type designs. Such bias overlooks the important feature that, quite often, experimental research plans do not render the best fit for learning important lessons regarding individuals' choices and behaviors. Nevertheless, to do the reviewed studies justice, we thoroughly assessed this body of literature and made use of previous systematic reviews to establish the MQS criteria tailored for this group of studies.

Despite these limitations, findings from this review can help inform and foster the delivery of successful CF carrier testing/ screening and educational programs. Although some of the factors identified in the reviewed studies are not amenable to change (e.g., parity), many of those identified frequently are factors that can be changed through educational means. For example, Hegwer et al. ${ }^{51}$ found that a 60 -minute carrier testing educational program can increase participants' knowledge. Poppelaars et al..$^{31}$ also proposed that education activities for the lay public via mass media campaigns and school education programs can reduce perceived barriers of participating in CF carrier screening programs. Thus, health education efforts aimed at both the lay public and clients can significantly assist the development of theoretically sound, pedagogically appropriate, and cost-effective CF carrier screening/testing programs. Such programs (delivered in clinical settings or public education/media outlets) can contribute not only to decreasing perceived barriers to CF carrier screening, but also to increasing CF-related knowledge among high-risk populations.

\section{References}

1. Decruyenaere M, Evers-Kiebooms G, Denayer L, Welkenhuysen M. Uptake and impact of carrier testing for cystic fibrosis. A review and a theoretical framework about the role of knowledge, health beliefs and coping. Community Genet 1998;1: 23-35.

2. Sorenson JR, Cheuvront B, DeVellis B, Callanan N, et al. Acceptance of home and clinic-based cystic fibrosis carrier education and testing by first, second, and third degree relatives of cystic fibrosis patients. Am J Med Genet 1997;70:121-129.

3. Delvaux I, van Tongerloo A, Messiaen L, Van Loon C, et al. Carrier screening for cystic fibrosis in a prenatal setting. Genet Test 2001;5:117-125.

4. Levenkron JC, Loader S, Rowley PT. Carrier screening for cystic fibrosis: test acceptance and one year follow-up. Am J Med Genet 1997;73:378-386.

5. Murray J, Cuckle H, Taylor G, Littlewood J, et al. Screening for cystic fibrosis. Health Technol Assess 1999;3:1-104.

6. Genetic testing for cystic fibrosis. National Institutes of Health Consensus Development Conference Statement on genetic testing for cystic fibrosis. Arch Intern Med 1999;159:1529-1539.

7. The American College of Obstetricians and Gynecologists, American College of Medical Genetics. Preconception and prenatal carrier screening for cystic fibrosis: Clinical and laboratory guidelines ed. Washington, DC: American College of Obstetricians and Gynecologists, 2001.

8. Committee on Genetics, American College of Obstetricians and Gynecologists. ACOG Committee Opinion. Number 325, December 2005. Update on carrier screening for cystic fibrosis. Obstet Gynecol 2005;106:1465-1468.

9. Decruyenaere M, Evers-Kiebooms G, Denayer L, Van den Berghe H. Cystic fibrosis: community knowledge and attitudes towards carrier screening and prenatal diagnosis. Clin Genet 1992;41:189-196.

10. Neiger R, Abuelo DN, Passero MA. Attitudes toward genetic testing for cystic fibrosis among college students. J Genet Couns 1992;1:219-226. 


\section{Chen and Goodson}

11. Wake SA, Rogers CJ, Colley PW, Hieatt EA, et al. Cystic fibrosis carrier screening in two New South Wales country towns. Med J Aust 1996;164:471-474.

12. Honnor M, Zubrick SR, Walpole I, Bower C, et al. Population screening for cystic fibrosis in Western Australia: community response. Am J Med Genet 2000;93:198-204.

13. Garrard J. Health sciences literature review made easy: the matrix method, 2nd ed. Sudbury, MA: Jones and Bartlett Publishers, 2006.

14. Cook DJ, Mulrow CD, Haynes RB. Systematic reviews: synthesis of best evidence for clinical decisions. Ann Intern Med 1997;126:376-380.

15. Davis S, Reynolds L. Genetic testing, screening and counseling - an overview. Genet Issues Ment Retard 1997;1:1-6.

16. Denayer L, Evers-Kiebooms G, De Boeck K, Van den Berghe H. Reproductive decision making of aunts and uncles of a child with cystic fibrosis: genetic risk perception and attitudes toward carrier identification and prenatal diagnosis. Am J Med Genet 1992;44:104-111

17. Fanos JH, Johnson JP. Barriers to carrier testing for adult cystic fibrosis sibs: the importance of not knowing. Am J Med Genet 1995;59:85-91.

18. Hartley NE, Scotcher D, Harris H, Williamson P, et al. The uptake and acceptability to patients of cystic fibrosis carrier testing offered in pregnancy by the GP. J Med Genet 1997;34:459-464.

19. Lafayette D, Abuelo D, Passero MA, Tantravahi U. Attitudes toward cystic fibrosis carrier and prenatal testing and utilization of carrier testing among relatives of individuals with cystic fibrosis. J Genet Couns 1999;8:17-36.

20. Henneman L, Kooij L, Bouman K, ten Kate LP. Personal experiences of cystic fibrosis (CF) carrier couples prospectively identified in CF families. Am J Med Genet 2002;110:324-331.

21. Mennie ME, Liston WA, Brock DJ. Prenatal cystic fibrosis carrier testing: designing an information leaflet to meet the specific needs of the target population. J Med Genet 1992;29:308-312.

22. Buhi E, Goodson P. Predictors of adolescent sexual behavior and intention: a theoryguided systematic review J Adolesc Health 2007;40:4-21.

23. Denzin NK. The research act: a theoretical introduction to sociological methods. Chicago: Aldine Publishing Company, 1970.

24. Shoemaker PJ, Tankard JJ, Lasorsa DL. How to build social science theories. Thousand Oaks, CA: Sage Publications, 2004.

25. Janz NK, Champion VL, Strecher VJ. The Health Belief Model. In: Glanz K, Rimer BK, Lewis FM, editors. Health behavior and health education. San Francisco, CA: John Wiley \& Sons, 2002:45-66.

26. Goodson P, Buhi ER, Dunsmore SC. Self-esteem and adolescent sexual behaviors, attitudes, and intentions: a systematic review. J Adolesc Health 2006;38:310-319.

27. Suther S, Goodson P. Barrier to the provision of genetic services by primary care physician: a systematic review of the literature. Genet Med 2003;5:70-76.

28. Fang CY, Dunkel-Schetter C, Tatsugawa ZH, Fox MA, et al. Attitudes toward genetic carrier screening for cystic fibrosis among pregnant women: the role of health beliefs and avoidant coping style. Womens Health 1997;3:31-51.

29. O'Connor B, Cappelli M. Health beliefs and the intent to use predictive genetic testing for cystic fibrosis carrier status. Psychol Health Med 1999;4:157-168.

30. Henneman L, Bramsen I, van der Ploeg HM, Ader HJ, et al. Participation in preconceptional carrier couple screening: characteristics, attitudes, and knowledge of both partners. J Med Genet 2001;38:695-703.

31. Poppelaars FA, van der Wal G, Braspenning JC, Cornel MC, et al. Possibilities and barriers in the implementation of a preconceptional screening programme for cystic fibrosis carriers: a focus group study. Public Health 2003;117:396-403.
32. Tambor ES, Bernhardt BA, Chase GA, Faden RR, et al. Offering cystic fibrosis carrie screening to an HMO population: factors associated with utilization. Am J Hum Genet 1994;55:626-637.

33. Durfy SJ, Page A, Eng B, Chang PL, et al. Attitudes of high school students toward carrier screening and prenatal diagnosis of cystic fibrosis. J Genet Couns 1994;3:141155.

34. Witt DR, Schaefer C, Hallam P, Wi S, et al. Cystic fibrosis heterozygote screening in 5,161 pregnant women. Am J Hum Genet 1996;58:823-835.

35. Harris H, Scotcher D, Hartley N, Wallace A, et al. Pilot study of the acceptability of cystic fibrosis carrier testing during routine antenatal consultations in general practice. Br J Gen Pract 1996;46:225-227.

36. Poppelaars FA, Henneman L, Ader HJ, Cornel MC, et al. Preconceptional cystic fibrosis carrier screening: attitudes and intentions of the target population. Genet Test 2004;8:80-89.

37. Clayton EW, Hannig VL, Pfotenhauer JP, Parker RA, et al. Lack of interest by nonpregnant couples in population-based cystic fibrosis carrier screening. Am J Hum Genet 1996;58:617-627.

38. Loader S, Caldwell P, Kozyra A, Levenkron JC, et al. Cystic fibrosis carrier population screening in the primary care setting. Am J Hum Genet 1996;59:234-247.

39. Melancon MJ, De Braekeleer M. Adolescents' attitude towards carrier testing for cystic fibrosis. Eur J Hum Genet 1996;4:305-306.

40. Lafayette D, Abuelo D, Passero M, Tantravahi U. Attitudes toward cystic fibrosis carrier and prenatal testing and utilization of carrier testing among relatives of individuals with cystic fibrosis. J Genet Couns 1999;8:17-36.

41. Williamson R, Allison ME, Bentley TJ, Lim SM, et al. Community attitudes to cystic fibrosis carrier testing in England: a pilot study. Prenat Diagn 1989;9:727-734.

42. Mitchell J, Scriver CR, Clow CL, Kaplan F. What young people think and do when the option for cystic fibrosis carrier testing is available. J Med Genet 1993 30:538-542.

43. Payne Y, Williams M, Cheadle J, Stott NC, et al. Carrier screening for cystic fibrosis in primary care: evaluation of a project in South Wales. The South Wales Cystic Fibrosis Carrier Screening Research Team. Clin Genet 1997;51:153-163.

44. Mennie ME, Gilfillan A, Compton ME, Liston WA, et al. Prenatal cystic fibrosis carrier screening: factors in a woman's decision to decline testing. Prenat Diagn 1993;13:807-814

45. Botkin JR, Alemagno S. Carrier screening for cystic fibrosis: a pilot study of the attitudes of pregnant women. Am J Public Health 1992;82:723-725.

46. Tatsugawa Z, Fox M, Fang C. Education and testing strategy for large-scale cystic fibrosis carrier screening. J Genet Couns 1994;3:279-289.

47. Melancon MJ, De Braekeleer M. Adolescents' attitude towards carrier testing for cystic fibrosis. Eur J Hum Genet 1996;4:305-306.

48. Cuckle H, Quirke P, Sehmi I, Lewis F, et al. Antenatal screening for cystic fibrosis. $\mathrm{Br}$ J Obstet Gynaecol 1996;103:795-799.

49. Mennie ME, Gilfillan A, Compton M, Curtis L, et al. Prenatal screening for cystic fibrosis. Lancet 1992;340:214-216.

50. Patrick DL, Beery WL. Measurement issues: Reliability and validity. Am J Health Promot 1991;5:305-310.

51. Hegwer G, Fairley C, Charrow J, KE O. Knowledge and attitudes toward a free education and Ashkenazi Jewish carrier testing program. J Genet Couns 2006;15: $61-70$. 